\title{
Liderazgo de Directores Noveles de Latinoamérica a través de las Metáforas: Chile, Colombia y México
}

\author{
Novice Principals in Latin America and their Leadership \\ through Metaphors: Chile, Colombia and Mexico
}

\author{
Felipe Aravena ${ }^{1 *}$ \\ Clelia Pineda-Báez ${ }^{2}$ \\ Gema López-Gorosave ${ }^{3}$ \\ José María García-Garduño ${ }^{4}$ \\ ${ }^{1}$ Pontificia Universidad Católica de Valparaíso, Chile \\ ${ }^{2}$ Universidad de La Sabana, Colombia \\ ${ }^{3}$ Universidad Autónoma de Baja California, México \\ ${ }^{4}$ Universidad Autónoma de la Ciudad de México, México
}

\begin{abstract}
El propósito de este estudio fue comprender cómo caracterizan el ejercicio de liderazgo escolar los directores noveles cuando ejercen por primera vez el rol en el contexto latinoamericano considerando tres países: Chile, Colombia y México. Para ello fue utilizado la metáfora como herramienta analítica. Cuando los directores expresan su experiencia en el cargo a través de metáforas es posible comprender creencias, valores, teorías y paradigmas que subyacen a las visiones y prácticas de liderazgo. Esto es útil para poder comprender sus auto-concepciones sobre su rol, el sistema y también las relaciones interpersonales con otros actores educacionales. A través de una aproximación de corte cualitativo, utilizando entrevistas semiestructuradas, los resultados indican que los directores noveles de este estudio utilizan metáforas para simbolizar las relaciones complejas con la comunidad, las familias, los sostenedores y fundamentalmente expresan las complejidades de ser director por primera vez. Las metáforas utilizadas por los directores principalmente reflejan las etapas de control y supervivencia propias de quien asume sin experiencia previa el liderazgo en una escuela. Esta investigación aporta en la región latinoamericana donde, a pesar de la cercanía geográfica y de compartir rasgos culturales relativamente similares, no se han realizado estudios comparados sobre la situación de los directores noveles.
\end{abstract}

Descriptores: Director de colegio; Liderazgo; Chile; Colombia; México.

This paper explored leadership metaphors used by novice school principals from three Latin American countries: Chile, Colombia and Mexico. When school principals used metaphors is possible to understand beliefs, values, theories and paradigms related to their visions and leadership practices. This is useful to comprehend principal's self-concept, relationship with the system and also with other educational actors. Based on a qualitative approach, and using semi-structured interviews, results indicate that the novice school principals use metaphors to symbolize complex relationships with teaching staff, parents and the school community. The metaphors employed are associated with control and survival stages that are typical of newly appointed leaders. Despite recent attention to education leadership in Latin America, cross-country comparative studies are scarce. This study is the first one to provide insights about novice principal leadership in Latin America based on the comparison of three different contexts.

Keywords: Principals; Leadership; Chile; Colombia; Mexico.

*Contacto: felipe.aravena@pucv.cl

ISSN: $1696-4713$

www.rinace.net/reice/

revistas.uam.es/reice
Recibido: $\quad 29$ de agosto 2019

$1^{\text {a }}$ Evaluación: 12 de noviembre 2019

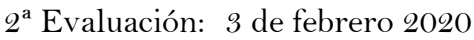

Aceptado: 1 de marzo 2020 


\section{Introducción}

Existe un volumen considerable de evidencia empírica y teórica que reporta al liderazgo como un factor clave en el mejoramiento escolar (Brock y Grady, 2012; Hitt y Tucker, 2016; Horng y Loeb, 2011). El liderazgo escolar es ejercido principalmente por los directores, quienes, al desplegar prácticas efectivas, impactan de manera positiva en las competencias docentes y en el aprendizaje de los estudiantes (Pashiardis y Johansson, 2016; Robinson, Hohepa y Lloyd, 2009). Para influir positivamente en la organización, los directores escolares requieren abordar desafíos multidimensionales que complejizan el rol (Sergiovanni, 1995) y que se agudizan cuando se es director sin experiencia (Bush y Jackson, 2002; Bush y Oduro, 2006; Northfield, 2013; Quong, 2006; Spillane y Lee, 2014).

En su mayoría, los estudios sobre directores noveles provienen del mundo anglosajón, con escasas contribuciones desde el contexto latinoamericano (Flessa et al., 2018). De hecho, en la literatura mundial solo el $0,34 \%$ de la producción investigativa corresponde a estudios latinoamericanos con temáticas de liderazgo y gestión escolar (Aravena y Hallinger, 2018). En Latinoamérica, entre los temas estudiados con mayor frecuencia están las características de los directores (Vaillant, 2011; Weinstein y Muñoz, 2012), las políticas y la profesionalización del director (Sandoval, 2015; Weinstein y Hernández, 2016; Weinstein et al., 2015) y descripciones sobre problemáticas que enfrentan los directores en sus contextos (García-Garduño, Slater y López-Gorosave, 2010; Montecinos, Bush y Aravena, 2018; Pineda-Báez et al., 2019). Pese a compartir rasgos culturales y socio-económicos, los estudios comparados sobre liderazgo escolar y en particular sobre la situación de los directores en Latinoamérica no son numerosos. En esta línea, se destaca el estudio realizado por Weinstein y Hernández (2016) donde se comparan las políticas de liderazgo en ocho sistemas escolares. Similarmente, Weinstein y colaboradores (2015) compararon programas de formación para líderes escolares en seis países de la zona y recientemente Silva y colaboradores (2017) analizaron desde un enfoque de liderazgo para la justicia social qué prácticas despliegan los directores cuando reciben a nuevos estudiantes en el caso de Costa Rica, México y también se agregó España. De la literatura investigativa en términos de liderazgo escolar en Latinoamérica no existen estudios comparados sobre los directores noveles. Esta investigación busca disminuir este vacío, contribuyendo a la comprensión de la compleja labor de los directores en Latinoamérica en sus primeros años de ejercicio profesional. El objetivo puntual fue comprender a través de las metáforas que emplean los directores noveles cómo se caracteriza el ejercicio del liderazgo en los primeros años en el puesto. Las preguntas que orientaron este trabajo fueron: ¿cuáles son las metáforas con las que los nuevos directores de Chile, Colombia y México reflejan su experiencia?, ¿cuáles son los problemas representados en dichas metáforas?, y ¿qué revelan las metáforas sobre su papel como líderes?

\section{Directores noveles de tres sistemas latinoamericanos: Contextualización y vacíos investigativos}

Para contribuir al vacío investigativo a continuación se presenta la situación de los directores noveles en cada uno de los países seleccionados para el estudio: Chile, Colombia y México. 


\subsection{Los directores noveles en Chile: Una situación de mejora fragmentada}

Existe consenso en la literatura nacional en establecer que la llegada de un nuevo director a una escuela es un momento crucial que puede aumentar o disminuir las posibilidades de mejora (Bellei, Vanni y Valenzuela, 2015; Weinstein y Muñoz, 2012). Asumiendo esta premisa, la política pública en Chile ha demostrado una orientación hacia el aseguramiento de la calidad del liderazgo y gestión escolar que ejercen los directores en sus respectivos contextos. Esta orientación se evidencia en cuatro iniciativas ministeriales: (1) en 2011 el Ministerio de Educación implementó un plan de formación de directores de excelencia totalmente financiado. Este plan ofrece anualmente a directores, docentes y otros profesionales de la educación, perfeccionamiento para impactar positivamente en los aprendizajes de los estudiantes; (2) en 2011, el sector municipal aplicó la Ley $\mathrm{N}^{\mathrm{o}}$ 20.501, la cual expresa que todos los directores deberán ser nombrados por el Servicio Civil y de la Alta Administración Pública asumiendo el cargo por un periodo de 5 años, sometiéndose a una postulación al cargo con más etapas de selección a través de un proceso más estandarizado a nivel nacional y más exigente a nivel profesional; (3) en 2015 se re-diseñó el Marco para la Buena Dirección y Liderazgo Escolar que estableció las competencias, prácticas generales y específicas y recursos personales que sirven para orientar la labor directiva; y (4) en 2017 se puso en marcha un programa impulsado por el Ministerio de Educación que ofrece un acompañamiento sistemático por parte de mentores (directores con más experiencia) durante 4 meses a directores noveles. Este programa, de carácter voluntario, se encuentra en una fase de piloto nacional siendo implementado en cinco regiones del país, para luego, a partir de la evaluación de la experiencia, implementarla a nivel nacional. Estas cuatro iniciativas ministeriales muestran que en Chile concurren esfuerzos por mejorar la situación directiva, sin embargo, las acciones no necesariamente se encuentran interconectadas, más bien se presenta una situación fragmentada. De ahí que en Chile no exista una carrera directiva que articule instancias de desarrollo profesional, evaluación y acompañamiento. Para eso la política pública requiere construir una arquitectura estratégica que coordine los esfuerzos por mejorar y apoyar sostenidamente la llegada al cargo de los directores noveles (Aravena, 2016).

El estudio sobre directores noveles en Chile es incipiente. Principalmente se focalizan en explorar los problemas que experimentan directores, sus trayectorias profesionales y sus necesidades formativas (Weinstein et al., 2016), así como también sus prácticas de liderazgo pedagógico (Leiva, Montecinos y Aravena, 2017), los cambios que buscan establecer (Galdames et al., 2018) y los problemas de sucesión escolar (Montecinos, Bush y Aravena, 2018). La literatura nacional coincide en señalar que ejercer el cargo de director en Chile es complejo porque, si bien se comparten desafíos globales, como por ejemplo: responder ante la emergencia y las múltiples demandas, la sensación de soledad, lidiar con el personal no competente, gestionar recursos, entre otros (García Garduño et al., 2011b), los directores noveles en Chile reportan que uno de los principales problemas es el ausentismo docente producto de la alta cantidad de licencias médicas (Weinstein et al., 2016). Ante este problema, los directores no cuentan con atribuciones para movilizar recursos hacia la contratación de otros docentes en el período que los estudiantes se encuentren sin profesor. Esto se agrava cuando las barreras burocráticas tienden a retrasar el proceso. Así, los directores noveles en Chile requieren balancear las demandas internas y externas con las expectativas y motivación personal dentro de uno de los sistemas latinoamericanos con más procesos de rendición de cuentas (Weinstein y Muñoz, 2012). 


\subsection{Directores noveles en Colombia: Intenciones de una política nacional de liderazgo escolar}

En Colombia, dos tipos de escalafón: el Estatuto Docente, Decreto Ley 2277 de 1979 (Ministerio de Educación Nacional-MEN, 1979) y el Nuevo Estatuto de Profesionalización Docente, Decreto Ley 1278 de 2002 (MEN, 2002), regulan los procesos de ingreso, permanencia, remuneración salarial, ascenso, formación y retiro de profesores y directivos en el ámbito oficial para los niveles de educación preescolar, básica y media. En el país se emplea el término 'directivo docente' para hacer referencia a cargos ejercidos por rectores, directores rurales, vicerrectores, coordinadores, directores de núcleos de desarrollo educativo y supervisores o inspectores de educación. Estas funciones son desempeñadas por educadores y normalistas superiores escalafonados, pero también pueden ser asumidas por profesionales de ámbitos distintos al educativo. En el sector público, la selección y nombramiento de cada uno de esos cargos se lleva a cabo mediante concurso abierto de méritos académicos y profesionales por la Comisión Nacional del Servicio Civil. Según el número de vacantes y las necesidades de cada región se expiden decretos que regulan y ponderan cada uno de los requisitos para el ingreso al concurso. El más reciente decreto (Comisión Nacional del Servicio Civil, 2017), estipula pruebas eliminatorias y clasificatorias en competencias básicas (comprensión lectora, redacción, cálculos numéricos básicos), pruebas psicotécnicas, valoración de antecedentes y una entrevista realizada por un experto en administración educativa y gestión. En el sector privado, el nombramiento del rector, o algún otro cargo de dirección o coordinación, es un proceso autónomo e independiente de cada institución educativa que es regulado por el Código Sustantivo del Trabajo y por normas concordantes con la carrera docente.

Recientemente, en Colombia se vislumbra un esfuerzo por orientar la formación de los directivos escolares hacia el liderazgo. Tal es el caso del programa Rectores Líderes Transformadores que ha sido reconocido como una experiencia innovadora en la formación de ese colectivo (Weinstein et al., 2015). La iniciativa es el resultado de sinergias entre el sector privado y el público. El programa es liderado por la Fundación Empresarios por la Educación (ExE), entidad privada sin ánimo de lucro que, con el aval de las Secretarías de Educación nacional, oferta el programa para directivos del sector público en aras de formarlos en competencias de gestión personal, pedagógica y administrativa y comunitaria.

La investigación sobre rectores en Colombia es casi inexistente. Los estudios realizados hasta el momento apuntan a que su formación se ha basado en teorías empresariales traducidas a la práctica, carentes de reflexiones sobre su articulación con los fines educativos y con los contextos de las instituciones (Camargo et al., 2007; Sandoval et al., 2008). Se señala también la ausencia de programas a nivel pregrado para formar profesionalmente a quienes aspiran a cargos directivos escolares en el país, en razón a que el decreto 272 de 1998 acabó con las licenciaturas en administración y supervisión educativa (Sandoval, 2015). Los rectores, por lo tanto, se cualifican en programas de posgrado ofrecidos por distintas universidades del país. De hecho, la más reciente caracterización de directivos escolares en Colombia indica que poseen un alto nivel de formación académica y que tienden a realizar estudios posgraduales en educación, no necesariamente en administración o gestión (Llanes et al., 2014). La oferta de preparación y formación del directivo escolar es altamente desregulada, como ocurre en otros países de la región (UNESCO, 2014). 
Un estudio reciente da cuenta de problemáticas que enfrentan los rectores en sus primeros años en el cargo y que refieren a las tensiones entre las demandas administrativas y académicas y a la complejidad de los contextos escolares en los que prevalecen dificultades de convivencia. Los estudios apuntan a la necesidad de formar en el liderazgo y al fortalecimiento del tejido relacional en las instituciones escolares (Pineda-Báez et al., 2019).

\subsection{Directores noveles en México: Investigación temprana y pérdida reciente del dinamismo}

El escenario de la dirección escolar en México experimentó cambios sustantivos en la presente década con la modificación del marco normativo y la implementación de nuevas políticas. En 2013 se reformó el Artículo $3^{\circ}$ Constitucional con la intención de garantizar una educación de calidad. Esta reforma trajo como consecuencia la creación de la Ley del Servicio Profesional Docente (LSPD) y de un Instituto Nacional para la Evaluación de la Educación (INEE). Con estas medidas se establecieron las nuevas condiciones de ingreso, promoción y permanencia para docentes y directivos de educación básica, así como un nuevo perfil para directivos compuesto de parámetros, rasgos e indicadores ideales para el puesto y susceptibles de evaluación. La primera vez que se evaluaron los cargos con funciones directivas mediante concursos de oposición fue al término del ciclo escolar 20152016.

Bajo este nuevo marco, el director que gana el concurso de oposición asume el puesto y está sujeto a un periodo de inducción de dos años en el que se prepara con cursos de liderazgo y gestión escolar. Al término de este periodo, el director accede a una evaluación del desempeño para ser nombrado director con permanencia si obtiene un resultado satisfactorio.

El interés por la investigación sobre la dirección escolar en México es anterior a las reformas arriba descritas, no obstante, la producción se estima escasa. García Garduño (1999) revisó la producción académica sobre el tema y esbozó cuatro etapas dentro de los trabajos de investigación sobre la dirección en el país. La primera se orienta hacia la teorización de la supervisión y organización escolar de la escuela rural mexicana iniciada en los años 30 del siglo pasado; la segunda etapa parte del estudio etnográfico sobre la eficacia de un director de escuela primaria publicado por Pastrana en 1997, al cual siguieron otras investigaciones que en su mayoría fueron tesis de posgrado que abordaron las características del director y supervisor escolar eficaz en los últimos años del siglo pasado. A principios de este siglo y con la implementación del Programa Escuelas de Calidad (PEC) en México, García Garduño identifica el surgimiento de una tercera oleada de estudios que abordan la dirección y gestión escolar en el marco del PEC, como los de Loera, Rangel y Contreras (2001) y Fierro (2006). Finalmente, el autor ubica una cuarta y última etapa de investigación sobre la dirección escolar en México generada por grupos de académicos participantes en dos redes internacionales dedicadas al tema: el International Successful School Principal Project (ISSPP), centrada en el estudio de la eficacia del director escolar, y el International Study of Principal Preparation (ISPP), abocada al estudio de los directores noveles de educación básica. Como parte del ISSPP se publicaron los estudios realizados por Torres-Arcadia y Flores-Kantisani (2012) y Torres-Arcadia y Pesqueira-Bustamante (2016) sobre la eficacia de los directores de educación media, y dentro de la ISPP los estudios realizados sobre directores noveles mexicanos de Slater, García-Garduño y López-Gorosave (2008), García-Garduño, Slater 
y López-Gorosave (2010, 2010b). Actualmente, la participación mexicana en las redes internacionales ha disminuido. Se sigue realizando investigación sobre la dirección escolar en México, aunque con menos dinamismo que en el pasado reciente.

\subsection{Metáforas}

Las metáforas son una forma de asignar sentido a la experiencia humana y de crear modelos simbólicos de las maneras de pensar y actuar (Lakoff y Johnson, 1980); son estructuras lingüísticas de representaciones cognitivas que ayudan a comprender ideas, conceptos, modelos y teorías para examinar, describir o analizar un problema (Bredeson, 1985; Gibbs, 1992). Estas estructuras suelen presentarse en imágenes mentales que dan cuenta de la compleja dinámica organizacional y el posicionamiento del "yo" de manera funcional e interpretativa en un contexto y tiempo limitado. Pueden ser expresadas de manera verbal, abierta, simbólica o bien ocultas en las estructuras mentales. De ahí que sean una oportunidad única para explorar los valores que sustentan las estructuras de pensamiento individual, relacional y organizacional (Martínez, Sauleda y Huber, 2001).

Las metáforas se mueven entre dos conceptos diferentes y complementarios: el comprender y el expresar. Por un lado, permiten comprender fenómenos complejos y abstractos a través de dispositivos menos complejos asociados a situaciones cotidianas, y por otro, permiten expresar el significado de una situación compleja a través de narrativas basadas en historias sociales construidas y comprendidas culturalmente (Cantón y Arias, 2010; Gibbs, 1992; Oberlechner y Mayer-Schönberger, 2003). Las metáforas hacen parte de los componentes simbólicos de las organizaciones escolares y desvelan además de la impronta cultural de la escuela, las ideologías de sus miembros y las formas de poder que se privilegian (Vázquez, 2007).

Las metáforas también han sido usadas como herramienta analítica para comprender las creencias, valores, teorías y paradigmas que subyacen a las visiones y prácticas de liderazgo (Heffernan, Netolicky y Mockler, 2019; Oberlechner y Mayer-Schönberger, 2003) y para comprender, los roles y las auto-concepciones sobre el desempeño de directores (Bullough, 1991). Entre los diversos estudios que conectan las metáforas y el liderazgo, la taxonomía propuesta por Bredeson (1985) plantea un marco pertinente para clasificarlas por cuanto las presenta en un continuo que se mueve de la inmediatez en la actuación, a la reflexión y visualización del futuro de la escuela. Su tipología incluye metáforas denominadas de mantenimiento, supervivencia y visión futura. La primera refiere a descripciones de tareas administrativas o actividades para mantener el flujo normal y buen funcionamiento de la escuela. Las segundas aluden a la superación de crisis y a respuestas asertivas a las necesidades más inmediatas del contexto, lo cual exige muchas veces posturas y acciones autocráticas. La visión futura por su parte, reúne metáforas centradas en una re-interpretación de la misión de la escuela y orientación de acciones hacia el futuro (p. 44).

Las metáforas explican también la influencia y el relacionamiento del director con otros actores de la escuela. Las investigaciones de Hernández-Amorós y Martínez-Ruiz (2018) y Cantón y Arias (2010) encontraron que las visiones de liderazgo de un grupo de directores se centraron en modelos individualistas basados en figuras de autoridad con motivaciones asociadas al deber y en modelos paternalistas con marcados rasgos patriarcales y heroicos de líderes que buscan la felicidad, el bienestar y la complacencia de su comunidad. Esto en contraposición con las tendencias actuales de modelos de liderazgo más colectivos o distribuidos. 
Las metáforas pueden tener un carácter dinámico. Es decir, pueden ayudar a comprender las trayectorias profesionales de los líderes, su transición de una situación a otra, sus etapas en el esclarecimiento y comprensión de situaciones y pueden explicar las dificultades para el logro del éxito, entre otros aspectos (Court y Ornan, 2007). Según Singh (2010) son susceptibles a cambios en los contextos y en el tiempo y parecen estar fuertemente influidas por políticas como por ejemplo la rendición de cuentas y los cambios producidos por reformas educativas (Schechter et al., 2018). Las metáforas en esta investigación se examinan para explicar las perspectivas de liderazgo de los directores, para determinar los sentidos que le asignan a su rol y a las problemáticas de sus contextos.

\section{Método}

Se llevó a cabo una investigación exploratoria comparada de corte cualitativo, fundamentada en el supuesto de que el cotejo entre dos o más objetos o sujetos es un proceso cognoscitivo, sistemático y deliberado que permite el descubrimiento de semejanzas y diferencias entre esos objetos o sujetos para avanzar en el conocimiento que se ha construido de ellos (Piovani y Krawczyk, 2017, p. 834). El estudio emplea entrevistas para comparar las metáforas empleadas por directivos noveles de 3 contextos Latinoamericanos para descubrir cómo caracterizan el ejercicio del liderazgo en los primeros años en el puesto. Los atributos comunes a los sujetos se desglosan en el siguiente apartado.

\section{Participantes}

Se llevó a cabo un muestreo intencional, es decir, una selección de sujetos que aportan información clave sobre el fenómeno que se estudia que para este proyecto fue el liderazgo en directores noveles. Participaron seis directivos de educación primaria que cumplieron con cuatro criterios: a) tener menos de dos años en el puesto, debido a que la literatura especializada establece este periodo como el más difícil (García Garduño et al., 2011a); b) dirigir una escuela de sostenimiento público; c) dirigir una escuela primaria y d) otorgar el debido consentimiento para el uso científico de la información proporcionada. Dos directivos son de Chile, dos de Colombia y dos de México; algunos datos generales de ellos y de sus escuelas se aprecian en el cuadro 1.

Cuadro 1. Datos generales de directores y escuelas participantes

\begin{tabular}{cccccccc}
\hline País & Edad & Experiencia & GÉnero & Nivel & Matrícula & N & ConteXto \\
\hline Chile & 35 & 10 meses & Femenino & Básico & 108 & 11 & Urbano \\
Chile & 34 & 14 meses & Masculino & Básico & 327 & 31 & Urbano \\
Colombia & 45 & 16 meses & Femenino & Básico & 890 & 53 & Urbano \\
Colombia & 60 & 12 meses & Masculino & Básico & 1200 & 65 & Urbano \\
México & 38 & 16 meses & Masculino & Básico & 215 & 13 & Urbano \\
México & 27 & 9 meses & Masculino & Básico & 142 & 10 & Urbano \\
\hline
\end{tabular}

Fuente: Elaboración propia.

En el caso de Colombia se seleccionaron rectores noveles, debido a que algunas de sus funciones son más parecidas a las de los directores en Chile y México. A pesar de que la muestra no es representativa, las prácticas de liderazgo de los participantes pueden, en cierta medida, ser representativas del marco de gestión escolar de los países mencionados. 


\section{Instrumentos y técnicas de recolección de datos}

Se diseñó un guion de entrevista semi-estructurada para explorar, entre otros aspectos, las motivaciones de los participantes para ser director, las dificultades y éxitos auto percibidos en su gestión, y las recomendaciones o sugerencias que darían a un nuevo director. Además, se solicitó a los entrevistados elaborar una metáfora que representara su experiencia como director novel. En la exploración de esa metáfora se inquirió por actores, lugares, formas de interacción, momentos críticos, acciones implementadas y su auto-imagen como líder.

\section{Procedimiento}

El guión de la entrevista fue diseñado de manera conjunta y piloteado en cada país para realizar los ajustes pertinentes. Un aspecto que intrigó a los investigadores fue si los directores serían capaces de describir su labor a través de una metáfora. El pilotaje demostró que fueron capaces de describir su experiencia como director novel por medio de esa figura. Una vez validado el instrumento, se llevaron a cabo las entrevistas procurando condiciones similares en los tres países: realizarla de forma individual, fuera del edificio escolar y en horarios distintos al del trabajo, con el fin de evitar interrupciones. Las entrevistas duraron aproximadamente 60 minutos cada una de ellas, fueron audio grabadas y posteriormente transcritas con el consentimiento de los participantes.

\section{Análisis de las metáforas}

El análisis individual consignó una matriz socializada entre los miembros del equipo investigador con un doble propósito: por una parte, complementar, comparar y contrastar las perspectivas de cada investigador y por otra, asegurar que la comprensión de las entrevistas estuviera libre de sesgos; estrategia que se conoce como triangulación por investigador (Okuda, y Gómez-Restrepo, 2005).

\section{Hallazgos: Las metáforas}

\subsection{Chile: En un bosque lleno de pajaritos}

Victoria asumió la dirección de la escuela en febrero de 2017. No contaba con experiencia en el sector público y venía de otra comuna. En su primer día de trabajo recibió la noticia de que su escuela sería sede de la inauguración del año escolar de toda la comuna. Ese día asistieron todas las autoridades políticas (Alcaldes, gobernadores y secretarios regionales de educación, entre otros). Para ella el primer día fue caótico y nunca pensó recibir tanta gente externa a la escuela. De forma que ese día fue su presentación ante toda la comunidad con la prensa incluida. Avanzados los meses, declara que como directora siente que la gestión del tiempo y la multiplicidad de demandas desde el nivel central son los principales obstaculizadores en su desempeño. "Es bien vertiginoso todo, siento que en el trabajo todo ocurre muy rápido, el tiempo se me hace muy poco, sobre todo por la carga de actividades adicionales de la administración central, siempre me siento corta por los tiempos. Siento que estoy como en un bosque lleno de pajaritos".

La escuela se encuentra inserta en un bosque, y la directora asume el rol de guarda parque. Porque “(...) yo (se refiere a ella) tengo que estar pendiente de todo... que no se me vaya ningún detalle, soy la responsable de aquí”. En este bosque, los pajaritos serían todos los actores de la escuela, cada uno de ellos alborota el espacio a causa de la "diversidad de personas que existen con diferentes habilidades”. En cambio, el sostenedor pareciera vivir 
fuera del bosque porque... "solo aparece en casos puntuales, es como una niebla que llega al bosque, solo de vez en cuando... no siempre está presente”. Como guarda parque, es central conocer el espacio natural del bosque porque "creo que es importante conocer bien el contexto y la comunidad de la escuela. Para mí ha sido fundamental saber con quienes trabajo, sus historias, porque igual yo vengo de afuera, soy nueva, yo no los conocía y ellos tampoco me conocían a mí, entonces ese proceso de conocimiento mutuo y de la cultura es importante".

\subsection{Chile: El laberinto con salida}

Andrés lleva un año y 2 meses como director. El día que llegó a la escuela el sostenedor organizó un acto para despedir al director anterior. El encuentro entre ambos fue frío, solamente hubo contacto cuando le entregó las llaves de la escuela. Después de recibir las llaves, abrió su oficina y se sentó sin saber por dónde comenzar. Relata que lo primero que pensó fue cómo el sostenedor organiza una ceremonia de despedida al otro director estando el mismo presente. De ahí en adelante tuvo que ganarse a los profesores que usualmente asistían a los paros y marchas llamadas por el colegio de profesores, perdiendo muchas horas de clases. Los mayores problemas que reporta en su trabajo están asociados a la falta de compromiso de la familia y a la gestión de recursos económicos por parte de los sostenedores. La escuela atiende a una población muy vulnerable donde las drogas y la violencia son parte común del escenario escolar. Por eso, su paso por la dirección se asemeja a estar en un laberinto. Un laberinto es un espacio de encrucijadas, complejo y confuso. Usualmente en los laberintos los caminos son múltiples y pueden llevar a confusiones y/o errores de salida. Se vuelve a empezar, perdiendo muchas veces todo lo avanzado. "En el laberinto, para mí, las murallas con las que te encuentras son dificultades, como por ejemplo la familia”. La familia participa de forma intermitente en el proceso de aprendizaje de los estudiantes y para Andrés, más que un facilitador se convierte en un obstaculizador de su gestión. "Los padres no logran entender que queremos que los niños salgan adelante y vienen con gritos o groserías que no corresponden".

Un director requiere observar la realidad contextual de la escuela "uno tiene que saber dónde meterse y cómo hacerlo, yo como sabía que tenía que estar presente...un director debe saber muy bien donde poner el primer pie y después el otro (dentro del laberinto)". Para Andrés, en sus primeros meses es importante mostrarse visible ante la comunidad por eso se tiene que caminar por el laberinto "yo como estoy en los recreos exijo que los profesores estén en los recreos, me doy vuelta por los baños así que están impecables, me doy vuelta por sector parvulario por eso nunca hay accidentes, un director tiene que estar presente, me doy vuelta por fuera, uno tiene que hacer liderazgo... tiene que ser omnipresente". Pese a los múltiples desafíos, el laberinto se evidencia como una etapa compleja pero superada "yo logré salir muy bien del laberinto".

\subsection{Colombia: El capitán de un barco}

Jaqueline, con menos de dos años como directora, usa esta metáfora con la intención de mostrar que es ella quien tiene el control total de la escuela. Así como el capitán asume el comando absoluto de un barco y es el responsable de todas sus maniobras en el mar: atracar, desatracar, manejar el personal y enfrentar tormentas, Jaqueline se asume como la autoridad para llevar a buen término los procesos académicos y administrativos de la escuela, que siempre van acompañados de un alto grado de incertidumbre: "Entonces tú zarpas. Sabes que tienes que llevarlos al final de año, digamos año por año, que los tienes que llevar. Pero tú de pronto en ese momento puedes perder el rumbo, o hay una tormenta, 
o cualquier cosa puede pasar en el camino". La incertidumbre y la adversidad se derivan de la alta vulnerabilidad social del contexto en el que se ubica la escuela. Jaqueline atiende cerca de 900 niños de grados cero a once que provienen de familias de escasos recursos económicos, con graves problemas de drogadicción y abandono. Aunque cuenta con experiencia como administradora y coordinadora en el sector público y privado, reconoce que el trabajo administrativo absorbe gran cantidad de su tiempo lo cual incide en su capacidad para atender el trabajo pedagógico: "nuestro trabajo es administrativo. La rectoría no es pedagógica. Nosotros necesitamos estar más tiempo en los colegios y los compromisos y la gestión y lo administrativo, lo fiscal no nos lo permite".

Así como el capitán de un barco enfrenta amotinamientos, Jaqueline enfrenta tensiones asociadas a las actitudes y compromiso de los profesores y a la presión del sindicato. De hecho, la escuela ha tenido una alta rotación de directivos; seis en los últimos tres años. Su reacción frente a esas tensiones fluctúa entre el autoritarismo y el acompañamiento que ella denomina pedagógico: “[actuar] mucho con la ley, pero suavemente. Mire profe, yo le voy a exigir a usted porque además de sus seis horas, yo, de vez en cuando le puedo decir que se quede dos horas para completar las ocho". El abrir espacios de diálogo ha sido importante para transitar de la autoridad a la concientización sobre el papel de los maestros en la escuela y para la generación de sentido de pertenencia.

\subsection{Colombia: Sembrar semillas}

Germán lleva un año como director en una escuela a la que asisten cerca de 1000 estudiantes y 65 profesores. La escuela está ubicada en el área urbana de Bogotá, en un contex to que requiere del apoyo y vigilancia de la policía nacional por tratarse de una zona de consumo y venta de estupefacientes. En la metáfora de Germán las semillas son los estudiantes y la siembra está referida a incentivar y fortalecer el carácter de los niños. La siembra significa aportarle al estudiante a través de un trabajo centrado en el desarrollo personal, principalmente en el fortalecimiento de la dimensión emocional y de valores como el respeto, la aceptación y valoración de la diferencia. Es posible que incentivar valores emane de su deseo de prevenir que los niños incurran en las prácticas nocivas que caracterizan sus contextos: "prevenir es mejor que curar porque nuestros niños están en una edad en la cual puede uno formar o aportarle algo más, cambiarle un hábito es más fácil en esta edad".

Los profesores hacen parte del proceso de siembra y aportan a la transformación de los estudiantes con su saber. Germán valora sus aportes y considera que forman parte de un equipo que debe trabajar en objetivos mancomunados: "Por naturaleza el rector es quien va dando una dirección. Pero el rector no debe estar solo. Debe tener su equipo en el cual cada uno ejerce una función". Su rol es de direccionamiento y se ve a sí mismo como la cabeza de su comunidad. "El rector sería, digamos, el caporal de la finca. Entonces en compañía de todos mis obreros. Humildemente el caporal es otro obrero. Pero mis obreros, -los docentes, y con mis obreros -niños, vamos a hacer ese cultivo y hacerlo crecer. El caporal lo define el diccionario de la Real Academia Española como la "persona que hace de cabeza de alguna gente y la manda". Tiene una posición de poder. En la metáfora Germán hace parte del equipo, pero usa el pronombre posesivo "mis" (obreros) para referir a su posición de mando. En general su visión como director es idealista y soñadora como él mismo reconoce: "Yo lo veo muy soñador. Pero tengo que también ser muy vencedor en el sentido de que tenemos que esforzarnos para trabajar todos en equipo y alcanzar ese ideal de nuestros sueños" 


\subsection{México: El engrane que lubrica y dirige}

Los engranes son empleados para transmitir potencia mecánica de un elemento a otro mediante movimientos giratorios o alternativos. Probablemente esa capacidad de potenciar elementos, fue la que hizo que Ángel concibiera este mecanismo como un símil de su función en la escuela. Él dirige con mucho entusiasmo una escuela primaria que atiende a 215 niños, la mayoría hijos de jóvenes padres trabajadores.

La escuela se ubica en la periferia de una pequeña ciudad del noroeste de México y cuenta con la infraestructura necesaria para su funcionamiento, aunque el director experimenta dificultades para el mantenimiento del edificio escolar. Dentro del personal hay siete profesores de grupo, dos de educación física, un asesor técnico pedagógico y un equipo de especialistas para el apoyo de la educación regular.

Con poco menos de dos años de experiencia directiva, Ángel piensa que "el director es como un engrane que facilita, apoya y se asegura de que las ayudas lleguen al aula y que impacten el aprendizaje". Desde su primer día en el puesto asumió el rol del engrane principal del sistema escolar, al enfrentar con éxito el caos generado por un accidente de una madre de familia, provocado por otra al salir de la escuela. Haber tomado las decisiones oportunamente movilizó al resto del personal y a las autoridades competentes para solucionar el imprevisto, lubricando de esta manera el funcionamiento de los componentes del mecanismo escolar y algunos extraescolares, y posicionándose casi de inmediato como el engrane principal de la escuela.

Con soltura explica cómo ser engrane principal: "busco recursos externos, cuestiono las políticas de gestión escolar, ejerzo liderazgo, defiendo la integridad de los niños y lucho por mejorar la escuela" ... También describe los elementos de su sistema de engranaje: "el aprendizaje de los niños, la capacitación de los maestros, el involucramiento de los padres de familia y la gestión ante las autoridades".

El engrane docente lo lubrica con capacitación y apoyo oportuno, este engrane lo combina con el del equipo de apoyo a la educación regular; "las nuevas tecnologías ayudan a mantener engranados a los padres de familia" y, estudioso de las normas internas y externas, acciona para engranar organizaciones públicas y privadas que engranen, finalmente, los niños al sistema. Desde su lógica de acción: "no sólo el engrane principal lubrica, también la colaboración y la capacitación docente, pero es el director el que jala las otras ruedas".

\subsection{México: El surfeador: Él y la ola}

Surfear es un deporte marítimo, quienes lo practican se suben en una tabla y conservan el equilibrio para desplazarse aprovechando las mejores olas. Brian, un director con nueve meses en el puesto, equipara su experiencia a la de un surfeador. Su entrada al puesto se enmarca por la resistencia magisterial ante los jóvenes directores dictaminados por concurso, y no por escalafón, como tradicionalmente se hacía.

La escuela dirigida por Brian tiene un profesorado que oscila entre los 35 y 62 años de edad, rango que contrasta con su juventud; se encuentra bien equipada y cuenta con un grupo de especialistas como apoyo a la educación regular. No obstante, los niveles de reprobación, de deserción y el contexto de violencia y drogadicción, hicieron que las autoridades la clasificaron como centro educativo de alto riesgo. 
Esta escuela se ubica en Ensenada, un puerto del Pacífico mexicano en el que se practica el surf, quizá por ello Brian equipara "la experiencia del personal, los vicios y la cultura magisterial" a una ola peligrosa que lo puede ahogar. Para sobrevivir, recurre a sus conocimientos como el surfer a la tabla que lo salva si sabe usarla en el momento adecuado: "veo a mis maestros y decido por dónde y cómo hacerle, si me equivoco, me arrastra (la ola) y no va a haber otra igual, en ese momento, no hay quien te ayude, el surfista está solo frente a la ola".

No sólo los maestros le representan olas. Las generadas por los niños parece sortearlas con facilidad: "durante el recreo me gusta platicar con ellos sobre cómo les va, qué les gusta hacer, a qué les gusta jugar, qué cosas les gustaría que fueran diferentes en la escuela”. Las olas levantadas por los padres de familia, si bien las sintió peligrosas cuando llegó, pudo atravesarlas con éxito manteniendo una comunicación asertiva y rindiendo cuentas puntualmente: "abrí espacios para que ellos estén en la escuela, no los aislé, escuché sus necesidades, y medié los problemas surgidos con docentes, soy muy claro con las finanzas, peso que entra a la escuela, peso que informo a la comunidad y a la sociedad de padres de familia". Sin duda, la ola más grande es "mediar los problemas con los profesores, creo que resolver los problemas con los padres o con los niños es más sencillo que resolver los problemas con los docentes".

Así como el dominio del surf requiere reflejos, equilibrio, conocimientos y habilidades, ejercer la dirección escolar requiere responder oportunamente, sopesar las acciones, conocer el contexto escolar y ser hábil comunicador, mediador y negociador: "no me he caído, he atravesado las olas, no me han arrastrado, estoy aprendiendo a montarme bien en la tabla para salir exitoso".

\section{Discusión}

El presente estudio exploró las metáforas empleadas por los directores noveles de Chile, Colombia y México, para simbolizar el ejercicio del liderazgo en los primeros años en el puesto. Las metáforas permiten discutir los resultados en tres niveles: problemas experimentados por los directores noveles, caracterización del rol directivo y la relación de las metáforas con ciertas características de los sistemas educativos y políticas publicas de los países estudiados.

Las metáforas empleadas por los participantes en esta investigación develan varios problemas comunes a los tres países estudiados. Un primer problema común es la capacidad de responder a la abrumadora carga de demandas externas. Esto conduce a los directores noveles a sentirse en espacios representados con imágenes que denotan temor o incertidumbre, como lo son el bosque denso y el laberinto. La preocupación por las multi-tareas relacionadas con la gestión también es señalada en el estudio de Schechter y colaboradores (2018) que muestra, además, que en las metáforas empleadas se soslaya el papel del directivo en el desarrollo profesional de los maestros para mejorar los procesos de aprendizaje de los estudiantes. Este aspecto también estuvo ausente en la mayoría de los casos indagados. Otro problema que simbolizan las metáforas corresponde a las tensiones que enfrentan los directores noveles con el personal ineficaz. Esto traslada a los directores a verse a sí mismos como guardabosques, caporales de finca y capitanes de barco sorteando tormentas o surfeadores enfrentando olas peligrosas. Esto, aunado a las dificultades para gestionar recursos económicos para el sostenimiento de la escuela, 
incrementa aún más la incertidumbre manifestada en metáforas como el laberinto o el bosque lleno de pajaritos. Los problemas referidos a la excesiva carga administrativa y a la consecuente necesidad de gestionar el tiempo para cumplir con todas las tareas, así como las dificultades con el personal docente y también gestionar el vínculo con las familias, han sido reportados con anterioridad en un estudio sobre los problemas de los directores noveles en Chile (Galdames et al., 2018; Montecinos, Bush y Aravena, 2018), Colombia (Pineda-Báez et al., 2019) y México (García-Garduño et al., 2010) y aquí se revelan como problemas comunes entre directores de los tres países.

Responder a estos problemas ha sido difícil para algunos directores, y aunque todos sobreviven con mayor o menor éxito a los desafíos contextuales, consideran fundamental conocer la especificidad del contex to de la escuela que se va a liderar. Para estos directores el conocimiento del contexto donde lideran es clave, muy probablemente por las dificultades experimentadas en su fase de ingreso al cargo y por otras relacionadas con la vulnerabilidad del contexto socio-económico en el que la escuela se inserta. Este último aspecto es muy evidente en el caso colombiano y mexicano, ya que los directores tienen a su cargo escuelas con un alto grado de vulnerabilidad, acosadas por problemáticas como el microtráfico de drogas, la pobreza, y la delincuencia. Por lo mismo sugieren a los aspirantes al puesto, priorizar el conocimiento de los maestros, de los padres y de la cultura de la escuela.

A través de las metáforas también los líderes fueron capaces de simbolizar su rol. La mayoría de los directores asumen un rol de omnipresencia. Se trata de directores para quienes el éxito de su labor depende de su capacidad individual para asumir el control absoluto sobre las personas y la organización en su conjunto, lo cual refleja inseguridades para delegar tareas. La incapacidad de poder delegar tareas a otros también fue uno de los problemas reportados por los directores noveles en estudios previos en México (LópezGorosave et al., 2010). Las metáforas del guardabosque y el capitán de un barco ejemplifican ese deseo de supervisión y control para llevar a buen término los procesos escolares. En otro caso, el director identifica su tarea como sembrador que espera que lo plantado fructifique. Su metáfora concentra fuertes rasgos del liderazgo paternalista descrito por Hernández-Amorós y Martínez-Ruiz (2018) en el que surgen características heroicas de protección de los miembros de la comunidad escolar. Pero este director equipara su papel con un caporal de finca, lo cual implica más allá del direccionamiento, manejo de poder y visualizar al personal como subalterno (Bredeson, 1985). Estos directores asumen y ejercen la autoridad formal controlando, en la medida de lo posible, a las personas y a la organización en su intento por desempeñarse de modo exitoso. Pareciera que mediar entre apoyo y control es un dilema para los directores noveles en los tres países estudiados.

Los directores noveles asumen el rol con un alto nivel de incertidumbre. Durante los primeros meses experimentan el temor de verse rebasados por el personal o atrapados por las circunstancias del entorno. En dos casos es notorio el esfuerzo realizado para asumir la autoridad. Por ejemplo, el director en un laberinto, emplea esta imagen para aludir a la complejidad contextual que lo atemoriza. No obstante, enfrenta los desafíos exigiendo la presencia de sus profesores durante los recreos, verificando que las instalaciones estén limpias y recorriendo la escuela para vigilar su buen funcionamiento. Estas prácticas reflejan el deseo de salir de la encrucijada a través del rol omnipresente descrito en líneas anteriores. Otro caso es el del director más joven, quien, al igual que un surfeador, teme que una ola lo rebase. La ola experimentada con mayor temor, quizá por enfrentarla solo, 
es la representada por los vicios y la cultura laboral del personal con muchos años en servicio, mismos que, a diferencia del joven director, ingresaron antes de que los concursos de oposición y la evaluación docente fuera parte de la política de calidad educativa en México. El sentimiento de soledad es compartido por otros directores poco apoyados por la autoridad superior o por quienes llegan de otra comunidad. Los directores que experimentan temor de verse perdidos o rebasados y, como consecuencia, de no llegar a ser buenos directores, comparten metáforas asociadas a "sobrevivir en el cargo" (Bredeson, 1985).

Otro caso que se destaca porque se aleja de la omnipresencia y la supervivencia implícita en varios de los relatos de los participantes, es el de la metáfora del engranaje. El logro en la cohesión de grupos de trabajo y en la fuerza que se le imprime a la estructura de la escuela parecen derivarse de la seguridad del director para superar obstáculos y de su claridad para trazar metas con rapidez en las que se moviliza a la comunidad interna y externa de la escuela. Este caso se caracteriza por su gestión de 360 grados, despliega un liderazgo integral centrado en el aprendizaje de los alumnos, interviene en el desarrollo profesional de los profesores, e involucra efectivamente a padres de familia y autoridades en la mejora de la escuela. Esta metáfora guarda rasgos similares a las de 'modelos o guías' propuesta por Hernández-Amorós y Martínez-Ruiz (2018) en las que prevalece la visión social del director como modelo y propulsor de la participación de la comunidad para el logro de los objetivos de la escuela y en la que se tiende a favorecer una visión de liderazgo más distribuido.

Al ser las metáforas esencialmente construcciones socio-culturales (Bullough, 1991; Court y Ornan, 2007; Martínez, Sauleda y Huber, 2001; Oberlechner y Mayer-Schönberger, 2003), se encuentran arraigadas a las particularidades de cada uno de los sistemas escolares. Pese a las múltiples convergencias, el caso de las metáforas en Chile presenta un problema distintivo no tan explícito en relación a las metáforas colombianas y mexicanas. En ambas metáforas chilenas, se visualiza al sostenedor municipal como una figura importante. Esta figura se conceptualiza como un obstaculizador en la labor directiva. Las metáforas expresan que la estructura municipal chilena y esencialmente la figura del sostenedor, son elementos que complejizan más el liderazgo para la mejora. Para los directores chilenos, los sostenedores no necesariamente apoyan con recursos y acompañamiento profesional en la socialización profesional del rol y específicamente en el cumplimiento de las metas estratégicas para la mejora escolar del establecimiento. Esto coincide con los resultados de Montecinos, Bush y Aravena (2018), quienes establecen que para los directores noveles en Chile es complejo desplegar prácticas efectivas de liderazgo a nivel intermedio, es decir "líder hacia arriba". Para alguien que no ha tenido experiencia previa, es más difícil negociar políticamente con el nivel municipal, porque significa negociar con la estructura del sistema más que con las personas que lo componen. Esta dificultad ha sido reportada por diversos estudios internacionales (Northfield, 2013) y en la estructura del sistema escolar chileno con un alto nivel de rendición de cuentas se agudiza aún más (Weinstein y Muñoz, 2012).

Para el caso colombiano se puede afirmar que aspectos como el control y la autoridad, implícitos en las metáforas empleadas, podrían estar asociados al tamaño de los establecimientos, y por consiguiente al alto número de estudiantes y docentes, ya que el directivo escolar tiene bajo su responsabilidad todos los niveles educativos de educación básica y media desde el grado primero a once (Pineda-Báez et al., 2019). En los casos estudiados, esta diferencia es notoria si comparamos los tamaños de los establecimientos 
de México y Chile con los colombianos. A esto se suma que las escuelas se ubican en sectores de alta vulnerabilidad derivada de problemáticas como la pobreza, el microtráfico de drogas y la delincuencia, entre otras, lo que lleva a plantear metáforas que implican un trabajo más centrado en "sembrar" esperanza en la comunidad escolar. Para observar los resultados de la siembra se requiere contar con tiempo. Las características del sistema colombiano permiten a los directores proyectar una metáfora más a largo plazo. Esto porque en Colombia una vez pasado el período de prueba, un director asume su cargo a término indefinido y puede establecer sus propuestas de mejora con mayor laxitud en términos temporales, que por ejemplo en Chile, donde el cargo se asume por medio de un concurso público por un período de cinco años. Pareciera ser que las metáforas de los directores en Chile no cuentan con una proyección a largo plazo de su ejercicio de liderazgo, a diferencia de los directores en Colombia, y en menor medida en México.

En el caso mexicano, a pesar de que ambos directores ingresaron al puesto por concurso de oposición, tienen menos de dos años en el puesto y trabajan en contextos similares, sus percepciones sobre la magnitud de los problemas, las formas de intervenir, el centro de sus acciones y el sentido de las metáforas utilizadas, son sensiblemente diferentes. Mientras el director más joven, con menos tiempo en el puesto, menor cantidad de alumnos y personal, percibe el ejercicio directivo como un espacio desafiante y amenazante por constantes olas que ponen en riesgo su subsistencia en el puesto. El otro director con más de 10 años de edad y de experiencia en el sistema educativo y más de un año como director que el primero, se percibe como un gestor capaz de hacer confluir las fuerzas internas y externas en beneficio de los alumnos, a pesar de los obstáculos que se presentan. Estas diferencias plantean interrogantes que merecería la pena explorar no solo en México: la importancia de la trayectoria personal y profesional de los nuevos directores en las percepciones y ejercicio de la dirección escolar, y la evolución de las percepciones de los nuevos directores en el primer y segundo año en el puesto. Esto último dado que se ha abordado la evolución de los directores entre el primer y tercer año en el puesto, no así entre los primeros dos (López-Gorosave et al., 2010).

Los hallazgos confirman lo que otros autores han encontrado a nivel internacional: es difícil que los directores sin experiencia desplieguen prácticas de liderazgo distribuido o de un liderazgo pedagógico de manera inmediata; la carga de la gestión escolar y los retos planteados por el contexto de la comunidad, son algunos factores que inciden en el predominio del liderazgo directivo de los nuevos directores (Northfield, 2013; Quong, 2006; Spillane y Lee, 2014). El conocimiento del contexto es vital para la supervivencia y éxito de los directores que asumen el cargo por primera vez. Este aspecto, el leer la realidad contextual, aunado a la carga administrativa, ocupan gran parte del tiempo y energía de los nuevos directores.

\section{Conclusiones}

Los hallazgos de este estudio permiten sostener que los directores noveles en Chile, Colombia y México pueden simbolizar su propia experiencia de liderazgo a través de las metáforas. Un primer grupo de metáforas simbolizan el ejercicio de liderazgo desde una perspectiva directiva o racional técnica (Hernández-Amorós y Martínez-Ruiz, 2018), como son los casos del capitán del barco (Colombia), caporal/sembrador de semillas (Colombia) y guardabosques (Chile). El segundo bloque de metáforas empleadas son de supervivencia (Bredeson, 1985), como el caso del surfeador (México) y del laberinto con 
salida (Chile). La única metáfora que revela el ejercicio de un liderazgo menos directivo es la dirección como engrane (México). En línea con los estudios de Berenson (1985) y de Hernández-Amorós y Martínez-Ruiz (2018), en este trabajo prevalecen los modelos de supervivencia, fuertemente asociados a la necesidad de afianzar la confianza para responder a la carga administrativa y a situaciones de relacionamiento con los miembros de la comunidad escolar. La excesiva carga de gestión y accountability, sumada a los retos planteados por el contexto en el que se inserta la escuela, dificultan la puesta en marcha de formas de liderazgo más compartido o propuestas de liderazgo pedagógico e intensifica la omnipresencia del directivo como herramienta de control. Esto porque en todas las metáforas los directores noveles se auto-perciben como una figura clave e indispensable en la mejora escolar. Concibiendo al director como el último responsable de todo lo que acontece en la escuela (Spillane y Lee, 2014).

En los tres países estudiados, las metáforas entendidas como una forma de asignar sentido a la experiencia de liderazgo, evidencian que liderar por primera vez una escuela es altamente complejo, estresante y demandante. Esto se refleja en la tipología de problemas reportados que se vinculan a características idiosincráticas de los sistemas latinoamericanos. Entre los principales problemas se cuentan: lidiar con múltiples demandas, tratar con personal ineficaz, establecer vínculos con las familias, dificultades para gestionar recursos económicos, excesiva carga administrativa, gestionar el tiempo personal, entre otros. Adicionalmente, las metáforas también expresan que estos problemas se traducen en una dimensión emocional reportando sensaciones de miedo, inseguridad y aislamiento en el cargo.

A la luz de las problemáticas expresadas por los directores en el contexto latinoamericano, emerge también la necesidad de fortalecer su formación profesional. Una formación centrada en desarrollar capacidades críticas y reflexivas para analizar el contexto de intervención de los directores noveles pareciera ser primordial. Esto implica proveer espacios de aprendizaje para identificar, indagar y observar qué factores contextuales abren oportunidades y generan barreras para la mejora escolar. A su vez, desafía a los programas de formación profesional a pensar en nuevas formas de apoyo y acompañamiento en el cargo para lograr amortiguar los altos niveles de inseguridad, miedo y estrés que reportan los directores latinoamericanos en su primera experiencia de liderazgo. La mentoría por un director experimentado con prácticas efectivas comprobables en contextos similares podría ser una estrategia de desarrollo profesional que debiese ser considerada de manera formal en cada uno de los sistemas escolares.

El propósito de la investigación se logró aportando a la literatura el primer estudio comparativo de la situación de los directores noveles en Latinoamérica. Sin embargo, se reconocen ciertas limitaciones. Una primera limitación es el carácter exploratorio de la investigación que centra la mirada en los problemas que experimentan los directores noveles. Esto permite sugerir posibles estudios que profundicen sobre las diversas estrategias que han sido efectivas para afrontar las adversidades propias del cargo. A su vez, los estudios exploratorios abren posibilidades de investigación futura. En este caso, el estudio sugiere el desarrollo de otros estudios locales y comparativos que amplíen los hallazgos presentados. Estudios que aborden la perspectiva de género en la experiencia de liderazgo, las trayectorias de desarrollo profesional de los directores noveles, y las prácticas de liderazgo asociados a los niveles de desempeño de las escuelas sirven para profundizar el conocimiento empírico sobre la situación de los directores en latinoamérica. También se reconoce como una segunda limitación del estudio el tamaño de la muestra, 
por lo que una segunda sugerencia es realizar estudios con un número mayor de participantes. Finalmente, una tercera limitación corresponde al componente geográfico. Solo se revisaron tres países latinoamericanos, por lo tanto los hallazgos no pueden ser generalizados. Ampliar el estudio a otras latitudes de la región latinoamericana sería valioso para reducir el vacío investigativo de algunos países en términos de liderazgo como lo son: Bolivia, Honduras, Perú, Panamá, Venezuela, entre otros. Con esto se incrementaría el conocimiento sobre los directores en relación a sus percepciones, prácticas y modelos de liderazgo para latinoamericanizar la investigación sobre liderazgo escolar.

\section{Referencias}

Aravena, F. (2016). Preparing school principals in the Chilean scenario: Lessons from Australia, England and the United States. Journal of Educational Administration and History, 48(4), 342357. https://doi.org/10.1080/00220620.2016.1210586

Aravena, F. y Hallinger, P. (2018). Systematic review of research on educational leadership and management in Latin America, 1991-2017. Educational Management Administration $\Xi^{\circ}$ Leadership, 46(2), 207-225. https://doi.org/10.1177/1741143217745882

Bellei, C., Vanni, X. y Valenzuela, J. P. (2015). Una mirada a los procesos de mejoramiento escolar en Chile. Santiago de Chile: Universidad de Chile.

Bredeson, P. (1985). An analysis of the metaphorical perspectives of school principals. Educational Administration Quarterly, 22(1), 29-50. https://doi.org/10.1177/0013161X85021001004

Brock, B. y Grady, M. (2012). The daily practices of successful principals. San Francisco, CA: SAGE. https://doi.org/10.4135/9781506335308

Bullough, R. (1991). Exploring personal teaching metaphors in pre-service teacher education. Journal of Teacher Education, 42(1), 43-51. https://doi.org/10.1177/002248719104200107

Bush, T. y Jackson, D. (2002). Preparation for school leadership: International perspectives. Educational Management Administration E' Leadership, 30(4), 417-29. https://doi.org/10.1177/0263211X020304004

Bush, T. y Oduro, G. (2006). New principals in Africa: Preparation, induction and practice. Journal of Educational Administration, 46(4), 359-375. https://doi.org/10.1108/09578230610676587

Camargo, M., Sandoval, L., Vergara, M., Quiroga, C., Pedraza, A. y Halima, F. (2007). El directivo docente hoy. Entre la teoría y la práctica, Chía, Universidad de La Sabana.

Canton, I. y Arias, A. R. (2010). Metáforas y teorías implícitas de los directores escolares. Educatio Siglo XXI, 28(1), 227-248.

Court, D. y Ornan, E. (2007). Metaphor and narrative in the development of qualitative researcher. Curriculum and Teaching, 22(1), 87-98. https://doi.org/10.7459/ct/22.1.06

Fierro, C. (2006), Conflictos morales en el ejercicio de la función directiva del nivel básico (Tesis doctoral). Departamento de Investigaciones Educativas, México.

Flessa, J., Bramwell, D., Fernández, M. y Weinstein, J. (2018). School leadership in Latin America 2000-2016. Educational Management Administration E Leadership, 46(2), 182-206. https://doi.org/10.1177/1741143217717277

Galdames, S., Montecinos, C., Campos, F., Ahumada, L. y Leiva, V. (2018). Novice principals in Chile mobilizing change for the first time: Challenges and opportunities associated with a 
school's readiness for change. Educational Management Administration $\mathcal{E}^{2}$ Leadership,46(2), 318-338. https://doi.org/10.1177/1741143217707520

García-Garduño, J. M. (1999). ¿Cuáles son las características de liderazgo del director y del supervisor de educación básica efectivo? Una revisión de la literatura internacional y la investigación generada en México. En A. Rivera, C. Aramburu, M. C. Ortega y C. Pérez (Comp.), Psicología educativa: Programas y desafíos en educación básica (pp. 109-124). Ciudad de México: Universidad Pedagógica Nacional.

García-Garduño, J. M., Slater, C. y López-Gorosave, G. (2010). El director escolar novel de primaria: Problemas y retos que enfrenta en su primer año. Revista Mexicana de Investigación Educativa, 15(47), 1051-1073.

García-Garduño, J. M., Slater, C. y López-Gorosave, G. (2011a). Beginning elementary principals around the world. Management in Education, 25(3), 100-105.

https://doi.org/10.1177/0892020611403806

García-Garduño, J. M., Slater, C. y López-Gorosave, G. (2011b). El director novel: Estado de la investigación y enfoques teóricos. REICE. Revista Iberoamericana sobre Calidad, Eficacia y Cambio en Educación, 9(3), 30-50.

Gibbs, R. (1992). Categorization and metaphor understanding. Psychological Review, 99(3), 572577. https://doi.org/10.1037/0033-295X.99.3.572

Heffernan, A., Netolicky, D. y Mockler, N. (2019). New and alternative metaphors for school leadership. Journal of Educational Administration and History, 51(2), 83-86.

https://doi.org/10.1080/00220620.2019.1585768

Hernández-Amorós, M. y Martínez-Ruiz, M. (2018). Principals' metaphors as a lens to understand how they perceive leadership. Educational Management Administration \& Leadership, 46(4), 602-623. https://doi.org/10.1177/1741143216688470

Hitt, D. y Tucker, P. (2016). Systematic review of key leader practices found to influence student achievement: A unified framework. Review of Educational Research, 86(2), 531-569. https://doi.org/10.3102/0034654315614911

Horng, E. y Loeb, S. (2010). New thinking about instructional leadership. Phi Delta Kappan, 92(3), 66-69. https://doi.org/10.1177/003172171009200319

Lakoff, G. y Johnson, M. (1980). Metaphors we live by. Chicago, IL: Chicago University Press.

Llanes, L., Duarte, P., Navarro, H. y Ramírez, Y. (2014). Estudio diagnóstico para determinar ¿̇quiénes son los rectores y directores de las instituciones educativas de la educación preescolar, básica y media en Colombia? Chía: Fundación Compartir y Fundación Escuela Nacional de Evaluación EValuar.

Leiva, V., Montecinos, C. y Aravena, F. (2017). Liderazgo pedagógico en directores nóveles en Chile: Prácticas de observación de clases y retroalimentación a profesores. Revista Electrónica de Evaluación Educativa, 22(2), 1-17. https://doi.org/10.7203/relieve.22.2.9459

Loera, A., Rangel, N. y Contreras, V. (2001). ¿Cómo transformar las escuelas? Lecciones desde la gestión escolar y la práctica pedagógica. Ciudad de México: SEP.

López-Gorosave, G., Slater, G. y García-Garduño, J. M. (2010). Prácticas de dirección y liderazgo en las escuelas primarias públicas De México. Los primeros años en el puesto. REICE. Revista Iberoamericana sobre Calidad, Eficacia y Cambio en Educación, 8(4), 32-49.

Martínez, M., Sauleda, N. y Huber, G. (2001). Metaphors as blueprints of thinking about teaching and learning. Teaching and Teacher Education, 17(8), 965-977.

https://doi.org/10.1016/s0742-051x(01)00043-9 
Ministerio de Educación Nacional. (1979). Estatuto docente. Decreto ley 2277 de 1979. Recuperado de http://www.mineducacion.gov.co/1621/articles-103879_archivo_pdf.pdf

Ministerio de Educación Nacional. (2002). Nuevo estatuto de profesionalización docente. Decreto Ley 1278 de 2002. Recuperado de http://www.mineducacion.gov.co/1621/articles86102_archivo_pdf.pdf

Montecinos, C., Bush, T. y Aravena, F. (2018). Moving the school forward: Problems reported by novice and experienced principals during a succession process in Chile. International Journal of Educational Development, 62, 201-208. https://doi.org/10.1016/j.ijedudev.2018.04.004

Northfield, S. (2013). The novice principal: Change and challenges. Canadian Journal of Educational Administration and Policy, 142, 158-182.

Pastrana, L. (1997). Organización, dirección y gestión en la escuela primaria: Un estudio de caso desde la perspectiva etnográfica, Ciudad de México: CINVESTAV.

Pashiardis, P. y Johansson, O. (2016). Successful school leadership: International perspectives. Londres: Bloomsbury. https://doi.org/10.5040/9781474270984

Piovani, J. I. y Krawczyk, N. (2017). Los estudios comparativos: Algunas notas históricas, epistemológicas y metodológicas. Educação छ Realidade, 42(3), 82 1-840.

https://doi.org/10.1590/2175-623667609

Pineda-Báez, C., Bernal-Luque, R. Sandoval-Estupiñan, L Y. y Quiroga, C. (2019). Challenges facing novice principals: A study in Colombian schools using a socialisation perspective. Issues in Educational Research, 29(1), 205-222.

Oberlechner, T. y Mayer-Schönberger, V. (2003). Through their own words: Towards a new understanding of leadership through metaphors. Boston, MA: Harvard University Press. https://doi.org/10.2139/ssrn.357542

Okuda, M. y Gómez-Restrepo, C. (2005). Métodos en investigación cualitativa: Triangulación. Revista Colombiana de Psiquiatría, 34(1), 118-124.

Quong, T. (2006). Asking the hard questions: Being a beginning principal in Australia. Journal of Educational Administration, 44(4), 376-88. https://doi.org/10.1108/09578230610676622

Robinson, V., Hohepa, M. y Lloyd, C. (2009). School leadership and student outcomes: Identifying what works and why. Wellington: New Zealand Ministry of Education.

Sandoval, L. (2015). Una experiencia innovadora en la formación de directivos escolares en Iberoamérica. Revista Iberoamericana de Educación, 69, 135-156. https://doi.org/10.35362/rie690147

Sandoval, L., Quiroga, C., Camargo, M., Pedraza, A., Vergara, M. y Halima, F. (2008). Necesidades de formación de directivos docentes: Un estudio en instituciones educativas colombianas. Educación y Educadores, 9(2), 12-48.

Schechter, C., Shaked, H., Ganon-Shilon, S. y Goldratt, M. (2018). Leadership metaphors: School principals' sense-making of a national reform. Leadership and Policy in Schools, 17(1), 1-26. https://doi.org/10.1080/15700763.2016.1232836

Sergiovanni, T. (1995). The principalship: A reflective practice perspective. Boston, MA: Allyn \& Bacon.

Silva, P, Slater, C., Lopez Gorosave, G., Cerdas, V., Torres, N., Antunez, S. y Briceno, P. (2017). Educational leadership for social justice in Costa Rica, Mexico, and Spain. Journal of Educational Administration, 55(3), 1-35. https://doi.org/10.1 108/JEA-03-2016-0033

Singh, K. (2010). Metaphor as a tool in educational leadership classrooms. Management in Education, 24(3) 127-131. https://doi.org/10.1177/0892020608090411 
Slater, C., García-Garduño, J. M. y López-Gorosave, G. (2008). Challenges of a successful firstyear principal in Mexico. Journal of Educational Administration, 46, 702-714. https://doi.org/10.1108/09578230810908299

Spillane, J. y Lee, L. (2014). Novice school principals's sense of ultimate responsibility: Problems of practice in transitioning to the principal's office. Educational Administration Quarterly, 50(3), 431-465. https://doi.org/10.1177/0013161X13505290

Torres-Arcadia, C. y Flores-Kastanis, E. (2012). All seem to need immediate attention. What should I do first? A successful principal in a new school assignment at Secundaria revolución. Journal of Cases in Educational Leadership, 15(3), 22-32. https://doi.org/10.1007/978-94-007-1350-5_32

Torres-Arcadia, C. y Pesqueira-Bustamante, N. G. (2016). Directores y directoras escolares en México. Casos de éxito. Recuperado de https://itunes.apple.com/us/book/directores-y-directorasescolares-en-m\%C3\%A9xico/id1112295251?mt=11\#

UNESCO. (2014). El liderazgo escolar en América Latina y el Caribe. Un estado del arte con base en ocho sistemas escolares de la región. Santiago de Chile: OREALC/UNESCO.

Vaillant, D. (2011). Improving and supporting principals' leadership in Latin America. En J. MacBeath y T. Townsend (Eds.), International handbook of leadership for learning (pp. 571585). Ámsterdam: Springer.

Vázquez, R. (2007). Las metáforas: Una vía posible para comprender y explicar las organizaciones escolares y la dirección de centros. REICE. Revista Iberoamericana sobre Calidad, Eficacia y Cambio en Educación, 5(3), 137-151.

Weinstein J. y Hernández, M. (2016). Birth pains: Emerging school leadership policies in eight school systems of Latin America. International Journal of Leadership in Education, 19(3), 241263. https://doi.org/10.1080/13603124.2015.1020344

Weinstein, J. y Muñoz, G. (2012). ¿Qué sabemos sobre los directores de escuelas en Chile? Santiago de Chile: Fundación Chile.

Weinstein, J., Cuellar, C., Hernández, M. y Flessa, J. (2015). Experiencias innovadoras y renovación de la formación directiva Latinoamericana. Revista Iberoamericana de Educación, 69, 23-46. https://doi.org/10.35362/rie69094

Weinstein, J., Cuellar, C., Hernández, M. y Fernández, M. (2016). Director(a) por primera vez. Un estudio sobre la experiencia y socialización de los directores noveles en establecimientos municipales en Chile. Revista Calidad en la Educación, 46(1), 12-45. https://doi.org/10.4067/S0718-45652016000100002

\section{Breve CV de los autores}

\section{Felipe Aravena}

Profesor e Investigador de la Pontificia Universidad Católica de Valparaíso, Chile. Ha publicado múltiples investigaciones sobre la situación de los directores escolares en Chile. Se encuentra actualmente coordinando programas de formación profesional para directores en el Centro de Liderazgo de Mejora Escolar de la misma Universidad. ORCID ID: https://orcid.org/0000-0002-1369-4190,Email: felipe.aravena@pucv.cl 


\section{Clelia Pineda-Báez}

Doctora en Educación por la Universidad del Sur de Illinois Carbondale. Becaria Fulbright. Profesora e investigadora de la Facultad de Educación de la Universidad de La Sabana, Colombia. Miembro activo del grupo "International Study of Teacher Leadership (ISTL)". Sus líneas de investigación incluyen liderazgo escolar, liderazgo docente, compromiso escolar, alfabetización en inglés como lengua extranjera. ORCID ID: https://orcid.org/0000-0002-1369-4190. Email: clelia.pineda@unisabana.edu.co

\section{Gema López-Gorosave}

Doctora en Ciencias Educativas en Universidad Autónoma de Baja California. Actualmente es miembro activo de la red "International Study of Leadership Development Network (ISLDN)". Sus líneas de investigación son: directores noveles, formación docente y liderazgo para la justicia social. ORCID ID: https://orcid.org/0000-0001-5324-1703. Email: gemagorosave@gmail.com

\section{José María García-Garduño}

Estudió un Doctorado en Educación en la Ohio University. Actualmente es profesor de tiempo completo en la Universidad Autónoma de la Ciudad de México. Ha sido también profesor de tiempo completo en las universidades: Iberoamericana, Autónoma de Estado de Morelos, Autónoma de Baja California, Autónoma del Estado de Hidalgo y Pedagógica Nacional. Sus intereses de investigación están relacionados con la dirección y organización escolar, la evaluación educativa y el curriculum. ORCID ID: https://orcid.org/OOoO0002-0955-0880. Email: josemariagarduno@gmail.com 